\title{
Treatment of human disease due to Mycobacterium bovis: a systematic review
}

\author{
To the Editor:
}

Mycobacterium bovis, a member of the Mycobacterium tuberculosis complex, is an important cause of disease in cattle, but it can also cause disease in humans [1]. Transmission to humans generally occurs after close contact with infected animals or consumption of unpasteurised contaminated dairy products $[1,2]$. The symptoms of human disease due to M. bovis are similar to those of disease caused by M. tuberculosis, although $M$. bovis is more likely to cause extrapulmonary disease [3]. In clinical practice, M. bovis can only be differentiated from M. tuberculosis using biochemical or genetic tests [3, 4].

Although human $M$. bovis disease is now rare in high-income countries, it is believed to be a more important health issue in low-income countries due to the lack of veterinary control measures and pasteurisation of milk [2,3]. It has been estimated that $M$. bovis accounts for $<1.5 \%$ of all human tuberculosis (TB) in regions outside Africa and $\sim 2.8 \%$ of all $\mathrm{TB}$ disease in Africa [5]. However, the incidence of $M$. bovis TB may be underestimated, because of the similarity of clinical features to TB caused by $M$. tuberculosis and because testing for M. bovis is not performed routinely.

As recommended by the United States Centers for Disease Control and Prevention, treatment of disease due to $M$. bovis usually consists of rifampicin, isoniazid and ethambutol [6]. Treatment duration is generally extended to 9 months due to the exclusion of pyrazinamide, since all strains of $M$. bovis are resistant to it. In the past two decades, while several reviews have investigated the epidemiology of $M$. bovis in humans $[2,5,7]$, no review has evaluated the treatment regimens and outcomes of disease due to $M$. bovis. Therefore, we conducted a systematic review of all published articles that reported drug regimens and results of treatment of human disease due to M. bovis.

The search was conducted in three electronic databases: MEDLINE (through OVID), Embase (through OVID) and the Cochrane Library, from the start date of each database until the date of the search (September 8, 2015). Medical subject heading terms and keywords (in title and abstract) related to M. bovis, drugs of interest and treatment outcomes were used for the search. The following inclusion criteria were used: 1) there was no language restriction in the search, but the full-text had to be in English, French, Chinese, Portuguese or Spanish; 2) M. bovis disease that was confirmed by genetic tests or biochemical tests, but not disease caused by $M$. bovis bacille Calmette-Guerin; 3) cohort or randomised trial; 4) end-of-treatment outcomes, as defined by the World Health Organization (WHO), were reported [8].

Study characteristics and treatment outcome information were abstracted, including country, study period, study participant number, age, sex, HIV prevalence, extrapulmonary TB, drug resistance pattern, drug regimen, treatment duration and end-of-treatment outcomes. End-of-treatment outcomes were recorded according to WHO categories: success (cure and complete), failure, death, loss to follow-up and relapse. Authors of the studies included were contacted to obtain additional information.

Due to differences in regimens, results were not pooled. The treatment success rate of each cohort was calculated as: success/(success + fail + relapse) or success/(success + fail + death + relapse + loss to follow-up).

985 publications were identified through the initial database search, of which 17 were selected for full-text review. Of these, 14 were excluded. Six did not describe treatment regimens, two were conference abstracts and one was in Russian; five reported regimens and outcomes but were excluded for different reasons, as follows. Cicero et al. [9] reported 6-month status, rather than end-of-treatment outcomes; KatARIA [10] and O'Donohue et al. [11] reported time to culture conversion; Esteban et al. [12] included multidrug-resistant $M$. bovis cases (seven out of 13) and used seven different regimens without reporting results by regimen; and SAURET et al. [13] reported treatment durations of isoniazid-rifampicin-ethambutol that varied from 4 to 12 months without stratifying results.

This left three studies which reported 439 patients with $M$. bovis disease from the USA, Argentina and the Netherlands, of whom 54.4\% were male, and median ages were 42 years, 45 years and 62 years, respectively (table 1). In the two studies that reported HIV status, 39 out of 74 subjects and eight out of 37 subjects tested were HIV-positive. More than half of the patients (59\%) had extrapulmonary disease, among whom lymph node disease was the most common form.

After excluding the patients who died, were lost or received other or unknown treatment regimens, two cohorts with 156 patients received the isoniazid-rifampicin regimen for 6-9 months and two cohorts with 113 patients 
TABLE 1 Summary of the demographic and clinical information, and end-of-treatment outcomes with isoniazid-rifampicin or isoniazid-rifampicin-ethambutol regimens in the three eligible studies

\begin{tabular}{|c|c|c|c|c|}
\hline & & First auth & or [ref.] & \\
\hline & LoBue [14] & CoRdova [15] & MAJOOR [16] & \\
\hline Country & USA & Argentina & The Netherlands & \\
\hline Study period & $1994-2003$ & $1996-2008$ & 1993-2007 & \\
\hline Method of confirmation & Biochemical & Biochemical & Genotype & \\
\hline Subjects & 167 & $39^{\#}$ & 231 & \\
\hline Males:females & $106: 61$ & 28:11 & $105: 126$ & \\
\hline Age years (median) & 42 & 45 & 62 & \\
\hline HIV (HIV+/tested) & $39 / 74$ & $8 / 37$ & NA & \\
\hline Extrapulmonary TB lonly or with pulmonary & $87 / 167$ & $10 / 39$ & $163 / 231$ & \\
\hline Major extrapulmonary form & N/A & Lymph node & Lymph node & \\
\hline Resistance to isoniazid or rifampicin \% tested & $7 \%$ isoniazid, $1 \%$ rifampicin & $3 \%$ rifampicin, $3 \%$ isoniazid-rifampicin & $5 \%$ isoniazid, $1 \%$ isoniazid-rif & fampicinף \\
\hline End-of-treatment outcomes & & & & \\
\hline Subjects in cohort & 167 & 23 & 110 & 81 \\
\hline Drug regimen & $\begin{array}{l}9 \text { months } \\
\text { isoniazid-rifampicin }\end{array}$ & $\begin{array}{l}\quad 8-12 \text { months } \\
\text { isoniazid-rifampicin-ethambutol }{ }^{\S}\end{array}$ & $\begin{array}{c}\text { Isoniazid-rifampicin }{ }^{f} \text { Isoniazid-rifampicin- } \\
\text { ethambutol }\end{array}$ & Others and unknown \\
\hline Success & $129 / 167$ & $14 / 23$ & $\begin{array}{l}25 / 40 \quad 91 / 110\end{array}$ & $35 / 81$ \\
\hline Failure & 0 & $1 / 23$ & $7 / 110$ & $6 / 81$ \\
\hline Death & $25 / 167$ & $5 / 23$ & $12 / 40$ & $25 / 81$ \\
\hline Loss to follow-up & $12 / 167$ & $3 / 23$ & $3 / 110$ & $15 / 81$ \\
\hline Relapse & $1 / 167$ & 0 & NA & NA \\
\hline Success rate (versus fail + relapse) $\%$ & 99 & 93 & 93 & \\
\hline $\begin{array}{l}\text { Success rate (versus fail + relapse + death + } \\
\text { loss to follow-up) \% }\end{array}$ & 77 & 61 & 83 & \\
\hline $\begin{array}{l}\text { Data are presented as } n \text { or } n / N \text {, unless otherwis } \\
\text { two received streptomycin-ethambutol-pyrazinan } \\
\text { in this study. Of the } 35 \text { patients tested, } 12 \text { ( } 5 \% \text { of } \\
\text { rifampicin in this study, individualised regimens } \\
\text { before } 2 \text { months. } f: 10 \text { out of } 40 \text { patients died wit } \\
\text { duration was } 6-9 \text { months ( } n=22), \geqslant 9 \text { months } \\
\text { isoniazid-rifampicin-pyrazinamide-ethambutol ar }\end{array}$ & $\begin{array}{l}\text { se stated. NA: not available. }{ }^{\#} \text { : } \\
\text { mide-ethionamide and died an } \\
\text { all patients) were resistant to } \\
\text { were used. }{ }^{\S} \text { : many patients re } \\
\text { thin } \leqslant 5 \text { months. Of the remain } \\
\text { ( } n=6) \text { and unknown ( } n=2 \text { ). } \\
\text { nd } 14 \text { received isoniazid-rifam }\end{array}$ & $\begin{array}{l}\text { no information on treatment outcomes wa } \\
\text { the other } 23 \text { received isoniazid-rifampicir } \\
\text { isoniazid, and two ( } 1 \% \text { of all patients) were } \\
\text { ceived pyrazinamide as well, but this was } \\
\text { ing } 30 \text { patients, one received isoniazid-rifa } \\
\text { \#\#: eight out of } 110 \text { patients died } \\
\text { icin-ethambutol; treatment duration was } 6\end{array}$ & $\begin{array}{l}\text { s available for } 14 \text { out of the } 39 \text { patients; of the } \\
\text { n-ethambutol. ?: the resistant patterns of } 196 \\
\text { resistant to isoniazid-rifampicin. }{ }^{+} \text {: for those } \\
\text { discontinued in all cases in which Mycobacteric } \\
\text { mpicin and } 29 \text { received isoniazid-rifampicin-p } \\
\text { within } \leqslant 5 \text { months. Of the remaining } 102 \\
6-9 \text { months }(n=69), \geqslant 9 \text { months ( } n=26 \text { ) and unkn }\end{array}$ & $\begin{array}{l}\text { e remaining } 25 \text { patients, } \\
\text { patients were unknown } \\
\text { resistant to isoniazid or } \\
\text { um bovis was diagnosed } \\
\text { oyrazinamide; treatment } \\
\text { patients, } 88 \text { received } \\
\text { nown }(n=7) \text {. }\end{array}$ \\
\hline
\end{tabular}


received the isoniazid-rifampicin-ethambutol regimen for 6-12 months (table 1). When the denominator for success rate included all poor outcomes (fail + death + relapse + loss to follow-up, which might be considered equivalent to an "intention to treat" analysis), success was $74 \%$ for the isoniazid-rifampicin regimen and $79 \%$ for the isoniazid-rifampicin-ethambutol regimen. When the denominator included only success + fail + relapse (equivalent to efficacy), success rates were $99 \%$ and $93 \%$ for the isoniazid-rifampicin regimen and the isoniazid-rifampicin-ethambutol regimen, respectively. The reasons for the differences between these two methods of calculation of success rate were the high death rates (overall 15\%) with rates of loss to follow-up contributing the remainder (6\%). The impact on treatment outcomes of 6 months' or 9 months' duration of use, or from the added use of ethambutol could not be estimated due to the limited number of studies and patients. The major reason for low success rates when all outcomes were considered was the high mortality in all three studies. In two studies (LoBue and Moser [14] and Cordova et al. [15]), this high mortality could have been due to the high rates of HIV co-infection in the patients.

The current study has several limitations, the most important of which is that only three studies could be identified for this review, reflecting the rarity of this condition, at least in settings where testing for M. bovis is performed routinely. In addition, we could not pool results across studies, due to differences in regimen and duration, allowing only a simple comparison of rates. Fluoroquinolones were used in only one study (MAjoor et al. [16]) and only for a small number of patients, preventing us from estimating results with these agents. The large proportion of patients treated for extrapulmonary disease meant that treatment completion, rather than cure, was measured in the majority of patients; this may have resulted in an overestimation of treatment success rate. An additional limitation was that some patients had underlying drug-resistant strains, which could have resulted in higher failure and mortality rates. HIV co-infection may also have contributed to the higher mortality rates, compounding the limitations in the interpretation of treatment outcomes with different treatment regimens.

In summary, there is very limited evidence supporting current recommendations for the treatment of human disease due to $M$. bovis. However, these limited data suggest that currently used regimens of isoniazid-rifampicin or isoniazid-rifampicin-ethambutol are adequate, although the benefit gained by adding ethambutol to isoniazid-rifampicin remains unclear. There were inadequate data to support a shorter duration of treatment of $<9$ months. Although better evidence to inform treatment recommendations for M. bovis would be welcome, a greater research priority would be to correct the paucity of accurate epidemiological and surveillance data in order to define the importance of $M$. bovis as a cause of human disease. disease due to $M$. bovis http://ow.ly/h92K3023fLu

Zhiyi Lan ${ }^{1}$, Mayara Bastos ${ }^{2}$ and Dick Menzies ${ }^{1}$

${ }^{1}$ Respiratory Epidemiology and Clinical Research Unit, Montreal Chest Institute, McGill University, Montreal, QC, Canada. ${ }^{2}$ Dept of Infectious Diseases, Federal University of Rio de Janeiro, Rio de Janeiro, Brazil.

Correspondence: Dick Menzies, Respiratory Epidemiology and Clinical Research Unit, Montreal Chest Institute, Room 419, 2055 Guy St, Montreal, Canada. E-mail: dick.menzies@mcgill.ca

Received: March 292016 | Accepted after revision: June 232016 | First published online: Aug 182016

Support statement: This work was funded by a grant from the World Health Organization, which received funding from the United States Agency for International Development. Funding information for this article has been deposited with the Open Funder Registry.

Conflict of interest: Disclosures can be found alongside this article at erj.ersjournals.com

Acknowledgements: The authors thank Philip A. LoBue (Division of Tuberculosis Elimination, Centers for Disease Control and Prevention, Atlanta, GA, USA), Christof J. Majoor (Academic Medical Center, Amsterdam, the Netherlands) and Ezequiel Cordova (Infectious Diseases Unit, Hospital Cosme Argerich, Buenos Aires, Argentina) for providing additional information.

\section{References}

Grange JM. Mycobacterium bovis infection in human beings. Tuberculosis 2001; 81: 71-77.

2 Cosivi O, Grange JM, Daborn CJ, et al. Zoonotic tuberculosis due to Mycobacterium bovis in developing countries. Emerg Infect Dis 1998; 4: 59-70.

3 Michel AL, Müller B, van Helden PD. Mycobacterium bovis at the animal-human interface: a problem, or not? Vet Microbiol 2010; 140: 371-381.

4 Grange JM, Yates MD, de Kantor IN. Guidelines for Speciation within the Mycobacterium tuberculosis Complex. 2nd Edn. Geneva, World Health Organization, 1996.

5 Müller B, Dürr S, Alonso S, et al. Zoonotic Mycobacterium bovis-induced tuberculosis in humans. Emerg Infect Dis 2013; 19: 899-908. 
6 American Thoracic Society, CDC, Infectious Diseases Society of America. Treatment of tuberculosis. MMWR Recomm Rep 2003; 52: 1-77.

7 de Kantor IN, Ambroggi M, Poggi S, et al. Human Mycobacterium bovis infection in ten Latin American countries. Tuberculosis 2008; 88: 358-365.

8 World Health Organization. Treatment of Tuberculosis Guidelines. 4th Edn. Geneva, World Health Organization 2010 .

9 Cicero R, Olivera H, Hernández-Solis A, et al. Frequency of Mycobacterium bovis as an etiologic agent in extrapulmonary tuberculosis in HIV-positive and -negative Mexican patients. Eur J Clin Microbiol Infect Dis 2009; 28: 455-460.

10 Kataria YP. Observations on human infection with Mycobacterium bovis. Tubercle 1969; 50: 14-21.

11 O'Donohue WJ Jr, Bedi S, Bittner MJ, et al. Short-course chemotherapy for pulmonary infection due to Mycobacterium bovis. Arch Intern Med 1985; 145: 703-705.

12 Esteban J, Robles P, Soledad Jiménez M, et al. Pleuropulmonary infections caused by Mycobacterium bovis: a re-emerging disease. Clin Microbiol Infect 2005; 11: 840-843.

13 Sauret J, Jolis R, Ausina V, et al. Human tuberculosis due to Mycobacterium bovis: report of 10 cases. Tuber Lung Dis 1992; 73: 388-391.

14 LoBue PA, Moser KS. Treatment of Mycobacterium bovis infected tuberculosis patients: San Diego County, California, United States, 1994-2003. Int J Tuberc Lung Dis 2005; 9: 333-338.

15 Cordova E, Gonzalo X, Boschi A, et al. Human Mycobacterium bovis infection in Buenos Aires: epidemiology, microbiology and clinical presentation. Int J Tuberc Lung Dis 2012; 16: 415-417.

16 Majoor CJ, Magis-Escurra C, van Ingen J, et al. Epidemiology of Mycobacterium bovis disease in humans, the Netherlands, 1993-2007. Emerg Infect Dis 2011; 17: 457-463.

\section{Faster for less: the new "shorter" regimen for multidrug-resistant tuberculosis}

To the Editor:

Multidrug resistant (MDR) and extensively drug-resistant (XDR) tuberculosis (TB) are growing clinical and public health concerns, with an estimated worldwide incidence and mortality of 480000 and 190000 cases, respectively (2014) [1]. The World Health Organization (WHO) End TB Strategy reiterates the MDR-/XDR-TB threat and the solutions to control the epidemic [2]. Unfortunately, large proportions of patients with resistant $\mathrm{TB}$ do not have access to adequate diagnostics and treatment yet, while treatment success rates remain suboptimal (as demonstrated in the largest retrospective cohort of MDR-TB patients, i.e., TB caused by Mycobacterium tuberculosis isolates resistant to at least isoniazid and rifampicin) and decrease further with resistance patterns beyond XDR-TB [3].

Presently, several of the available drugs have limited efficacy, being either toxic or unobtainable or both, and the treatment may take up to 24 months or longer. Although a few, new and repurposed drugs are fortunately available, clinicians often have difficulties in designing effective regimens [4], due to lack of drugs and rapid diagnostics, susceptibility results, comorbidities, drug toxicities and tolerability.

Recently, WHO published new recommendations aimed at speeding up TB second-line drug resistance detection (rapid molecular MTBDRsl test) and improving treatment outcomes of MDR-TB cases (shorter MDR-TB regimen) [5]. This is a demonstration of the efforts urgently being made to provide wider access to diagnosis and treatment in countries with the highest burden of MDR-TB. WHO has highlighted the advantages of the new regimen (consisting of 4-6 months of kanamycin, moxifloxacin, prothionamide, clofazimine, pyrazinamide, high-dose isoniazid and ethambutol followed by 5 months of moxifloxacin, clofazimine, pyrazinamide and ethambutol), providing a fact sheet with the necessary explanations. They include its shorter duration (9-11 months), which will improve adherence and its "relatively" low cost $(<1000$ US dollars per patient), which will ensure sustainability; these features are extremely important in resource-limited settings and in rich countries. It is possible that the antibiotic regimen may be modified: for example kanamycin is likely to be replaced by capreomycin or amikacin and these modifications may increase the overall cost of the regimen [6].

The regimen is recommended for MDR-TB cases not resistant to, and never treated with second-line anti-TB drugs and, therefore, should not be used if there is a documented or suspected resistance or previous use of one of the drugs composing the regimen [5]. The new push towards broader molecular testing at an earlier stage will enable patients to be selected for the shorter MDR-TB regimen more readily 